\title{
Estudio de las condiciones de emprendimiento en Morona Santiago a través de un modelo basado en informes del Global Entrepreneurship Monitor
}

\section{Study of the entrepreneurship conditions in Morona Santiago through a model based on reports from the Global Entrepreneurship Monitor}

\author{
Gelinek Haylis Zabala Guzmán ${ }^{1}$ *, Kevin Jhoshua Morquecho Salto ${ }^{1}$ y Dalmari Carolina Revelo Rivadeneira ${ }^{1}$ \\ ${ }^{1}$ Universidad Católica de Cuenca, Sede Macas \\ *ghzabalag94@est.ucacue.edu.ec
}

DOI: https://doi.org/10.26871/killkana_social.v2i3.335

\begin{abstract}
Resumen
La presente investigación explora las condiciones bajo las cuales se desarrollan los emprendimientos de la región norte de Morona Santiago, Ecuador, correspondiente a los cantones Huamboya, Pablo Sexto y Palora. Esta investigación tiene el carácter de exploratorio, el cual se fundamenta en el desarrollo y la aplicación de un modelo para el estudio del emprendimiento basado en los informes desarrollados por el Global Entrepreneurship Monitor, y el análisis de una muestra de 280 emprendimientos en referencia a 15 empresas de la región. Los factores evaluados tomados para este modelo corresponden a las áreas: Gubernamental, Educación, Cultura, Tecnología, Infraestructura Física, Infraestructura Comercial y Capital Humano. Estos factores fueron evaluados a través de una entrevista direccionada que consta de 29 preguntas en formato escala de Likert y ocho preguntas politómicas de selección única. Los resultados de la investigación están expresados en índices por región y por área económica conforme a la Clasificación Industrial Internacional Uniforme. Dichos indicadores expresan que los emprendimientos de la región estudiada poseen fuertes limitaciones por parte del área Gubernamental en cuanto a programas y políticas para con el emprendimiento, mientras que en lo Educativo los establecimientos de estudio primario, secundario y de tercer nivel no aportan con los conocimientos y valores necesarios para el desarrollo del emprendimiento. De manera similar, existe un acceso limitado a proveedores, consultores y tecnologías de calidad. Los antecedentes mencionados permiten concluir que muchos de estos emprendimientos no logran convertirse en empresas y por lo tanto requieren del desarrollo de nuevas estrategias mediante la cooperación multilateral entre entidades público-privadas o dentro de procesos comunitarios.
\end{abstract}

Palabras clave: condiciones, emprendimiento, modelo.

\begin{abstract}
The present investigation explores the conditions under which the entrepreneurship of the northern region of Morona Santiago, Ecuador, corresponding to the Huamboya, Pablo Sexto and Palora cantons, are developed. This research has the character of exploratory, which is based on the development and application of a model for the study of entrepreneurship based on the development of Global Entrepreneurship Monitor, and the analysis of a sample of 280 businesses in reference to 15 companies of the region. The evaluated factors considered for this model correspond to the following areas: Government, Education, Culture, Technology, Physical Infrastructure, Commercial Infrastructure, and Human Capital. These factors were evaluated through a directed interview that consisted of 29 questions in Likert scale format and eight polytomous questions of single selection. The results of the research are expressed in indexes by region and by economic area according to the International Standard Industrial Classification. These indicators show that the ventures of the studied region have strong limitations on the part of the Government in terms of programs and policies for entrepreneurship, while in the educational sector, primary schools, high schools and universities do not contribute with the knowledge and values necessary for the development of entrepreneurship. Similarly, there is limited access to suppliers, consultants and quality technologies. The background concludes that many of these ventures fail to become companies and therefore require new strategies through multilateral cooperation among private-public entities.
\end{abstract}

Key words: conditions, entrepreneurship, model . 


\section{Introducción}

Este estudio realizado en la región Norte de Morona Santiago-Ecuador dado que en la región no existe información suficiente acerca del emprendimiento, Motivo por el cual el presente estudio toma como referente el proyecto GEM para estudiar las condiciones del emprendimiento mediante un modelo que permita visualizar de manera integral los aspectos y situaciones en que estos se ven involucrados para de esta forma, en el sector público desarrollar políticas adecuadas para solucionar, corregir y fortalecer. De la misma forma en el sector privado poseer información para desarrollar estrategias para aprovechar ventajas y sortear obstáculos.

En la región de estudio se evaluó aspectos importantes para el desarrollo del emprendimiento, como son: Gobierno, Educación-Cultura, Tecnología, Infraestructura Física-Comercial, Capital Humano. Los cuales componen parte esencial del modelo que se busca desarrollar para de esta forma poseer información detallada y poder visualizar de forma integral el contexto en que se desarrollan los emprendimientos, para de esta forma poder dar solución a problemas o fortalecer ventajas.

\section{Marco Teórico}

\subsection{El modelo GEM}

El proyecto GEM el cual es de importancia internacional estudia las características de los emprendimientos mediante varias herramientas, entre las cuales se encuentran: Encuesta a Población Adulta y Encuesta a Expertos (Amorós, 2011).

Para efectos del presente estudio se tomó en consideración el formato de la Encuesta dirigida a expertos la cual consiste selecciona 36 expertos de cada país tanto del sector público como el privado, quienes evalúan 9 condiciones importantes del ecosistema emprendedor o "entrepreneurial framework conditions" (EFC) las cuales son: financiamiento para el emprendimiento, políticas y programas gubernamentales, educación emprendedora, transferencia de $\mathrm{I}+\mathrm{D}$, infraestructura profesional y comercial (Serida, Guerrero, Alzamora Ruiz, Borda, y Morales, 2017).

\subsection{Importancia de las condiciones del ecosistema em- prendedor $(E F C)$}

\subsubsection{Políticas y Programas Gubernamentales}

Las Políticas gubernamentales, son aquellos proyectos diseñados por la administración del Estado con la finalidad de tratar ciertas problemáticas de la sociedad (Alzate, 2011), para ello es necesaria la modificación de la estructura económico-política; sin embargo, si esto no es posible, se convertirán en un fracaso, pudiendo incluso agravar la situación inicial (Gavilanes, 2010). El objetivo por el cual son creadas las políticas de Estado son el apoyo, fomento y crecimiento del sector micro empresarial por medio del crecimiento económico y solidario; con esto, se pretende incorporar a los sectores menores y apoyar a las industrias nacientes para que puedan llegar a ser competitivas (Salinas, Machado, Ordoñez, y Aquino, 2016), Los programas gubernamentales cuentan con ayudas financieras destinadas al apoyo de los emprendedores tales como el del código de producción, el cual brinda incentivos tales como la exoneración por cinco años al pago del Impuesto a la Renta a empresas destinadas a la manufactura de productos esencialmente importados, mientras que se brindan deducciones al mismo impuesto a empresas destinadas a investigación y desarrollo tecnológico.

\subsubsection{La infraestructura física, comercia y profesional}

El Acceso a infraestructura física se refiere a la facilidad con la cual se puede llegar a recursos tales como telecomunicaciones, servicios por parte del sector público o privado a un precio considerable y asequible (Amorós y Abarca, 2014), por otro lado, el cumplimiento de las metas previstas, se ve afectado de gran manera en contar con las infraestructuras necesarias. En un emprendimiento son elementos claves para poder ejercer la actividad de la empresa la infraestructura comercial y profesional puesto que son colaboradores esenciales; la infraestructura comercial y legal hace referencia a los todos los servicios de carácter legal, administrativo y comercial como a la existencia de instituciones encargadas de promover el nacimiento negocios (Abarca, Espinoza, Mandakovic, Rojas, y Yañez, 2016). Los Indicadores globales muestran a América Latina con un débil desempeño logístico; teniendo una escasa inversión en infraestructura, manteniendo matrices de flotas antiguas (Abad, 2015).

\subsubsection{Indicadores socio-económicos que influyen en el emprendimiento}

La importancia de los indicadores socioeconómicos es fundamental para poder valorar los programas de los gobiernos, y así analizar si se están cumpliendo las metas y los objetivos fijados por las políticas públicas, también son importantes y necesarios poder realizar una evaluación y poder dar seguimiento, así se podrá predecir las tendencias que acometan a una un país, región, provincia o hasta un municipio todo esto con relación a la cuestión económica y social; es importante tener en cuenta múltiples variables, las cuales representan tanto factores externos como el PIB, cambios inflacionarios o tasas de interés; tanto como aspectos institucionales como leyes, sistemas fiscales, regulaciones; sin olvidar aspectos personales como nivel educativo, edad, género (Barrado y Molina, 2015).

\subsubsection{Educación, formación emprendedora y Transferen- cia de $I+D$}

Las personas en sí, tienen el deseo y la aspiración de emprender puesto que presentan esfuerzos por crecer, por superarse, por destacar; es por todo ello esencial estimular el emprendimiento puesto que no basta con los esfuerzos ejercidos en la adolescencia o en la adultez es necesario 
ejercer presencia desde la niñez. Para que la educación despierte el espíritu emprendedor del estudiante es necesario romper paradigmas y modelos pedagógicos donde el educando sea responsable de su propio aprendizaje. (Gutiérrez, 2011). Fortalecer el espíritu emprendedor es aprender a razonar de manera centralizada y analítica en los objetivos deseados, aprovechando las oportunidades siempre y cuando sean medidos de forma equitativa los riesgos, para todo ello es indispensable dar valor a los conocimientos presentes en economía, contabilidad y administración puesto que son estas áreas de conocimiento las cuales proporcionan datos indispensables para el éxito. La transferencia de Conocimiento y tecnología hace hincapié en el conocimiento producido en centros de investigación, los cuales permiten transferir discernimiento científico y tecnológico. (Donneys González y Blanco Campins, 2016). Esto resulta útil para todos los participantes: Las empresas pueden obtener una fuente de creatividad, dando innovación y oportunidades competitivas; los centros de investigación pueden conseguir datos importantes; la sociedad se beneficia con conocimientos ayudando al desarrollo económico.

\subsubsection{Apertura del mercado}

El comercio intenta mantener el ritmo que marcan los cambios experimentados por los consumidores induciendo de manera paralela modificaciones para orientar a los clientes hacia su producto (Ripol, 1995) esta constante renovación de ideas permite apoyar los nuevos negocios. Entre estas, destaca una ley llamada antimonopolio, la cual rige en contra de las conductas anticompetitivas que se presentan por medio de prácticas injustas en los negocios, como tal es el caso del monopolio (Aragón, Reyes, y Flores, 2017).

\subsubsection{Financiamiento}

Para la creación y operación de una empresa, es imprescindible el financiamiento.

Difícilmente los emprendedores cuentan con los montos necesarios, por lo que se ven obligados a buscar fuentes de financiamiento alternas. En Ecuador, la fuente más usual de financiamiento para las PYMES, son créditos bancarios o fondos propios (León, 2015). Ban Ecuador otorga financiamiento a empresas productivas con grandes facilidades de pago; La corporación financiera nacional también se suma con financiamientos a emprendimientos; Inversionistas Ángeles. Son aquellos inversores que ayudan con la inversión de empresas.

\subsubsection{Normas Sociales y Culturales}

Las normas sociales, son aquellas bajo las cuales se rige las decisiones, actividades y aspectos comportamentales de cada individuo en la sociedad. La cultura empresarial, por su parte, ayuda a la correcta interacción entre individuos, dando equidad, tranquilidad y libertad a todos y cada uno de los que conforman la empresa creando así un ambiente de trabajo adecuado (Proaño, 2014).

\subsubsection{Recursos Humanos}

Hoy en día los recursos humanos son muy valorados, puesto que pueden marcar gran diferencia entre el éxito o el fracaso; sin embargo, encontrar a las personas correctas no siempre es sencillo, pues existen ciertos criterios a valorar tanto por el empleador como por los candidatos a empleados (de Soto, 2006).

\section{Metodología}

\subsection{Modelo de la Investigación}

El presente trabajo es de carácter descriptivo donde se estableció un modelo para el estudio del emprendimiento, el cual utilizó como herramienta de recolección de datos: una entrevista direccionada a los emprendedores de varias áreas económicas conforme la Clasificación Industrial Internacional Uniforme (CIIU), la entrevista está compuesta de 29 preguntas en formato de escala de Likert y 8 preguntas polinómicas de selección única, distribuidas de la siguiente manera:

Tabla 1 Composición de la entrevista

\begin{tabular}{|c|c|c|}
\hline \multicolumn{2}{|c|}{ Aspectos Informativos (8 preguntas polinómicas y 2 preguntas de Likert) } \\
\hline \multicolumn{2}{|c|}{ Indicadores (28 preguntas de Likert) } \\
\hline $\begin{array}{c}\text { Políticas } \\
\text { Gubernamentales (4 } \\
\text { p) }\end{array}$ & $\begin{array}{c}\text { Programas } \\
\text { Gubernamentales } \\
(3 p) .\end{array}$ & Mercado interno (4p). \\
\hline $\begin{array}{c}\text { Educación y } \\
\text { Formación } \\
\text { Emprendedora (3p). }\end{array}$ & $\begin{array}{c}\text { Normas Sociales y } \\
\text { culturales (2p). }\end{array}$ & $\begin{array}{c}\text { Transferencia de I+D } \\
(3 p) .\end{array}$ \\
\hline Capital Humano (3p). & $\begin{array}{c}\text { Infraestructura } \\
\text { Comercial y } \\
\text { Profesional (3p). }\end{array}$ & $\begin{array}{c}\text { Infraestructura física } \\
\text { y de servicios (3p). }\end{array}$ \\
\hline
\end{tabular}

Para la interpretación de los datos y la determinación de los índices es necesario reconocer la siguiente escala de Likert:

Tabla 2 Escala de Valoración

\begin{tabular}{|c|c|c|c|}
\hline Calificación & $\begin{array}{c}\text { Escala } \\
\text { Numérica }\end{array}$ & Max & Min \\
\hline Muy alto & 4 a 2 & 4,000000 & 2,000001 \\
\hline Alto & 2 a 0 & 2,000000 & 0,000001 \\
\hline Medio & 0 & 0,000000 & 0,000000 \\
\hline Bajo & -1 a -2 & 0,000001 & 2,000000 \\
\hline Muy bajo & -2 a -4 & 2,000001 & 4,000000 \\
\hline $\begin{array}{c}\text { Ineficiente o datos } \\
\text { inexistentes }\end{array}$ & -4 a -6 & 4,000001 & 6,000000 \\
\hline
\end{tabular}

Para el cálculo de los índices correspondientes a los cantones y el general se realizó un promedio ponderado conforme a la distribución presentada. 
Tabla 3 Composición de los Resultados

\begin{tabular}{|c|c|c|c|}
\hline Indicadores & \multicolumn{3}{|c|}{ Distribución } \\
\hline $\begin{array}{l}\text { Área de } \\
\text { Estudio }\end{array}$ & \multicolumn{3}{|c|}{ Resultado General } \\
\hline \multirow[t]{2}{*}{ Cantones } & Pablo XI & Huamboya & Palora \\
\hline & \multicolumn{3}{|c|}{ Agricultura, ganadería, silvicultura y pesca } \\
\hline \multirow{9}{*}{$\begin{array}{l}\text { Áreas } \\
\text { Econó- } \\
\text { micas } \\
\text { (CIIU) }\end{array}$} & \multicolumn{3}{|c|}{$\begin{array}{l}\text { Comercio; actividades de reparación de vehículos } \\
\text { automotores y motocicletas. }\end{array}$} \\
\hline & \multicolumn{3}{|c|}{ Actividades de alojamiento y servicio de comidas } \\
\hline & \multicolumn{3}{|c|}{ Construcción } \\
\hline & \multicolumn{3}{|c|}{ Transporte, almacenamiento } \\
\hline & \multicolumn{3}{|c|}{ Servicios científicos, profesionales y técnicos } \\
\hline & \multicolumn{3}{|c|}{ Industrias manufactureras } \\
\hline & \multicolumn{3}{|c|}{ Actividad inmobiliaria } \\
\hline & \multicolumn{3}{|c|}{ Servicios de apoyo o administrativo } \\
\hline & \multicolumn{3}{|c|}{$\begin{array}{l}\text { Actividades de la salud y asistencia social; Enseñanza; } \\
\text { Información y Comunicación }\end{array}$} \\
\hline
\end{tabular}

\subsection{Población}

Para la determinación de la muestra se tomó como referente la base electrónica de datos de RUCs del Servicio de Rentas Interna (SRI), para filtrar la información se estableció las siguientes condiciones:

1) Estado de RUC activo

2) Estado del establecimiento abierto.

3) El emprendimiento tenga su origen en los Cantones de Huamboya, Pablo Sexto y Palora.

4) No sea de carácter público.

5) No sea trabajador bajo relación de dependencia.

6) De acuerdo a la codificación CIIU no corresponda a ninguno de estos sectores (esta discriminación de los sectores se dio por que la mayoría de estas actividades están bajo control público, otros sectores son bajo relación de dependencia y en el caso de lo financiero todas las entidades no cumplen el punto 3 que se menciona en este apartado):

- (B) Explotación minera.

- (D) Servicio de electricidad, gas, vapor y aire acondicionado.

- (E) Servicio de agua; alcantarillado, gestión de desechos y actividades de saneamiento.

- (K) Actividad financiera y de seguros.

- (O) Administración pública y defensa; planes de seguridad social de afiliación obligatoria.

- (T) Actividades de hogares; actividades como productor de bienes y servicios para uso propio.

- (U) Organizaciones y órganos extraterritoriales.

- (R) Actividades en relación de dependencia sector privado

- (S) Actividades en relación de dependencia sector publico

- (V) No realizan actividad económica - CIIU

Conforme a lo ya mencionado anteriormente la distribución de la muestra se basa en 1928 RUCs que representan la actividad emprendedora dando como resultado 297 individuos como muestra, para quienes mediante el muestreo aleatorio estratificado con afijación proporcional ,se distribuyó a los respectivos estratos establecidos en 10.

Tabla 4 Distribución de la muestra

\begin{tabular}{|c|l|c|}
\hline CIIU & \multicolumn{1}{|c|}{ Actividad Económica } & $\begin{array}{c}\text { Muestra } \\
\text { por } \\
\text { Estrato }\end{array}$ \\
\hline A & Agricultura, ganadería, silvicultura y pesca & 115 \\
\hline G & $\begin{array}{l}\text { Comercio; actividades de reparación de vehículos } \\
\text { automotores y motocicletas. }\end{array}$ & 73 \\
\hline I & Actividades de alojamiento y servicio de comidas & 24 \\
\hline F & Construcción & 23 \\
\hline H & Transporte, almacenamiento & 19 \\
\hline M & Servicios científicos, profesionales y técnicos & 15 \\
\hline C & Industrias manufactureras & 10 \\
\hline L & Actividad inmobiliaria & 7 \\
\hline P,Q,J & Servicios de apoyo o administrativo & 4 \\
\hline N & $\begin{array}{l}\text { Actividades de la salud y asistencia social; Ense- } \\
\text { ñanza; Información y Comunicación }\end{array}$ & 7 \\
\hline Total & \multicolumn{2}{|l}{} \\
\hline
\end{tabular}

\section{Resultados}

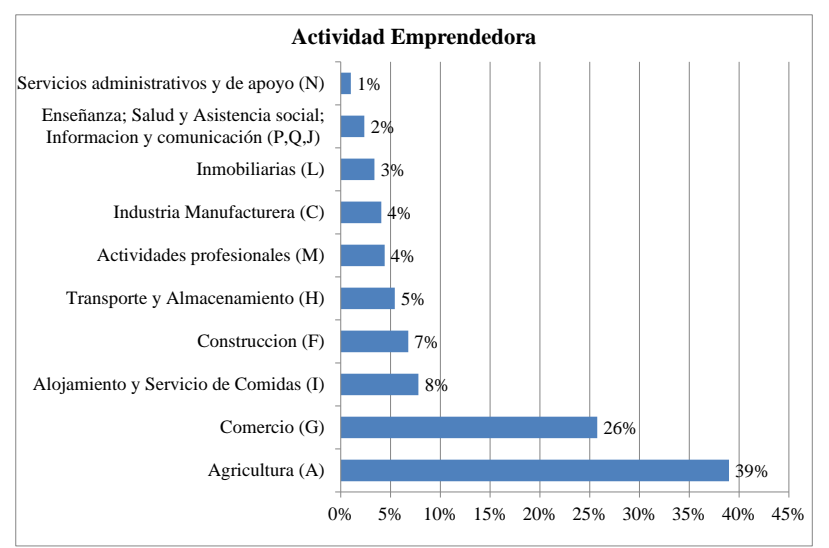

Figura 1. Composición de la Actividad Emprendedora

Fuente: Base de RUC del SRI a 10 de abril del 2018

Para un análisis más específico se dividió en dos grupos los sectores, el primero que corresponde a los 5 sectores que mayor actividad realizan en la región, y los 5 sectores que menos actividades realizan. El primer grupo se conforma de los sectores: Agricultura, Comercio, Alojamiento y Servicio de comidas, Construcción, Transporte y Almacenamiento, los cuales conforman el $85 \%$ de la actividad de la región, estos sectores son los que deben ser modernizados, fortalecidos y foco de atención de políticas públicas y centro de interés del sector privado.

El segundo grupo está conformado por: Actividades profesionales y científicas; Industria Manufacturera; Inmobiliarias; (Para este sector se unieron las siguientes áreas dada la poca actividad que estos representaban: Enseñanza; Salud y Asistencia social; Información y comunicación); Servicios administrativos y de apoyo. Estos en su totalidad representan un $15 \%$ de la actividad de la región, estos sectores deben ser promovidos en la medida que los anteriores se mejoren sus condiciones y se fortalezcan 


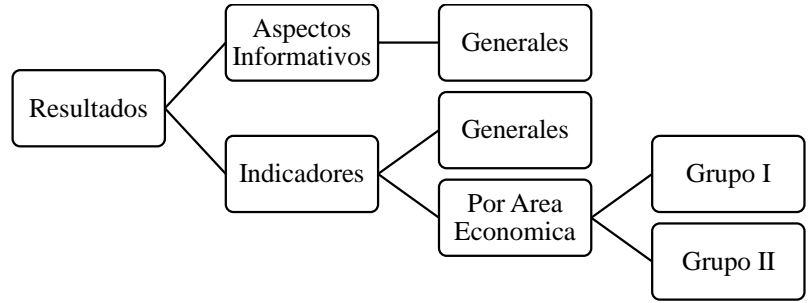

Figura 2. Distribución de Resultados

Fuente: Base de RUC del SRI a 10 de abril del 2018

Tabla 6 Aspectos Informativos

\begin{tabular}{|c|c|c|c|}
\hline \multicolumn{2}{|l|}{ Asesoría } & \multicolumn{2}{|c|}{ Cantidad trabajadores } \\
\hline Tributario & $68 \%$ & $0-5$ & $91 \%$ \\
\hline Ninguno & $19 \%$ & 11 a mas & $5 \%$ \\
\hline Contable & $6 \%$ & $6-10$ & $4 \%$ \\
\hline Otro & $6 \%$ & \multicolumn{2}{|c|}{ Administrador } \\
\hline Marketing & $1 \%$ & Dueño & $96 \%$ \\
\hline \multicolumn{2}{|c|}{ Tiempo de vida } & Otro & $3 \%$ \\
\hline 1-2 años & $31 \%$ & Profesional & $1 \%$ \\
\hline 3-5 años & $31 \%$ & Genero & \\
\hline 11 a mas & $20 \%$ & Femenino & $56 \%$ \\
\hline 6-10 años & $18 \%$ & Masculino & $44 \%$ \\
\hline \multicolumn{4}{|c|}{ Nivel de Estudio } \\
\hline Primario & $24 \%$ & Universitario & $23 \%$ \\
\hline Secundario & $51 \%$ & Postgrado & $1 \%$ \\
\hline & & Otro Estudios & $1 \%$ \\
\hline \multicolumn{2}{|c|}{ Edad primer negocio } & \multicolumn{2}{|c|}{ Edad actual } \\
\hline $18-25$ & $49 \%$ & $18-25$ & $5 \%$ \\
\hline $26-34$ & $38 \%$ & $26-34$ & $28 \%$ \\
\hline $35-42$ & $9 \%$ & $35-42$ & $36 \%$ \\
\hline 42 en adelante & $4 \%$ & 42 en adelante & $31 \%$ \\
\hline \multicolumn{2}{|c|}{ Uso de Financiamiento } & & \\
\hline Prestamos de Terceros & $\overline{1}, 03$ & & \\
\hline Capital Propio & 1,75 & & \\
\hline
\end{tabular}

Tabla 7 Indicadores Generales de la Región

\begin{tabular}{|c|c|c|c|}
\hline \multicolumn{2}{|l|}{ Gobierno } & \multicolumn{2}{|l|}{ Mercado Interno } \\
\hline $\begin{array}{l}\text { Apoyo al emprendimien- } \\
\text { to }\end{array}$ & $-1,64$ & $\begin{array}{l}\text { Cambio de Precio para } \\
\text { los Bienes-Servicios de } \\
\text { consumo }\end{array}$ & 0,06 \\
\hline $\begin{array}{l}\text { Regulaciones no son ba- } \\
\text { rrera para el emprendi- } \\
\text { miento }\end{array}$ & 1,12 & $\begin{array}{l}\text { Cambio de Precio para } \\
\text { los Bienes-Servicios de } \\
\text { consumo para empresas }\end{array}$ & $\overline{-}-$ \\
\hline $\begin{array}{l}\text { Políticas favorecen } \\
\text { la creación de de } \\
\text { emprendimiento }\end{array}$ & $-0,78$ & $\begin{array}{l}\text { Accesibilidad a nuevos } \\
\text { mercado por parte de } \\
\text { nuevas empresas }\end{array}$ & $\overline{-}$ \\
\hline $\begin{array}{l}\text { Efectividad de Burocra- } \\
\text { cia }\end{array}$ & 1,7 & $\begin{array}{l}\text { Efectividad de la ley anti- } \\
\text { monopolio }\end{array}$ & $\overline{0}-45$ \\
\hline $\begin{array}{l}\text { Eficiencia Profesionales } \\
\text { Parte Gubernamental }\end{array}$ & $-0,17$ & \multicolumn{2}{|l|}{ Educación } \\
\hline $\begin{array}{l}\text { Programas para el em- } \\
\text { prendimiento }\end{array}$ & $-1,75$ & $\begin{array}{ll}\text { Espíritu } & \text { Empresarial } \\
\text { Educación } & \text { Primaria y } \\
\text { Secundaria } & \\
\end{array}$ & 0,32 \\
\hline \multicolumn{2}{|l|}{ Cultura } & $\begin{array}{l}\text { Presencia de las Univer- } \\
\text { sidades }\end{array}$ & $\overline{2}, 41$ \\
\hline Valor del éxito individual & 0,03 & $\begin{array}{l}\text { Formación en dirección, } \\
\text { gestión de empresas }\end{array}$ & $\overline{2}-56$ \\
\hline $\begin{array}{l}\text { Estímulo a la Creativi- } \\
\text { dad, Riesgo empresarial e } \\
\text { Innovación }\end{array}$ & $-0,27$ & & \\
\hline
\end{tabular}

Tabla 6 Indicadores Generales de la Región...(Continuación)

\begin{tabular}{|l|l|l|l|}
\hline \multicolumn{2}{|c|}{ Tecnología } & \multicolumn{2}{c|}{ Capital Humano } \\
\hline $\begin{array}{l}\text { Acceso a nuevas investi- } \\
\text { gaciones y tecnologías }\end{array}$ & $-1,16$ & $\begin{array}{l}\text { Capacidad de Contratar } \\
\text { el Capital Humano nece- } \\
\text { sario }\end{array}$ & 1,33 \\
\hline $\begin{array}{l}\text { Acceso a tecnologías me- } \\
\text { diante ayuda guberna- } \\
\text { mental }\end{array}$ & $-1,95$ & $\begin{array}{l}\text { Capital Humano capaci- } \\
\text { tado }\end{array}$ & 1,68 \\
\hline $\begin{array}{l}\text { Transferencia de cono- } \\
\text { cimiento Universidades- } \\
\text { Empresa }\end{array}$ & $-1,52$ & $\begin{array}{l}\text { Reducción de contrata- } \\
\text { ción por políticas labora- } \\
\text { les }\end{array}$ & - \\
\hline \multicolumn{2}{|c|}{ Infraestructura física } & Infraestructura comercial y profesional \\
\hline $\begin{array}{l}\text { Apoyo de la infraestruc- } \\
\text { tura física }\end{array}$ & 1,79 & $\begin{array}{l}\text { Accesibilidad a consulto- } \\
\text { res, proveedores, subcon- } \\
\text { tratistas de calidad }\end{array}$ & 0,2 \\
\hline $\begin{array}{l}\text { Fácil accesibilidad a tele- } \\
\text { comunicaciones y servi- } \\
\text { cios básicos de calidad }\end{array}$ & 1,26 & $\begin{array}{l}\text { Accesibilidad a servicios } \\
\text { bancarios }\end{array}$ & 0,41 \\
\hline $\begin{array}{l}\text { Costo de acceder a Servi- } \\
\text { cios de telecomunicacio- } \\
\text { nes y servicios básicos }\end{array}$ & 1,01 & $\begin{array}{l}\text { Costo de acceder consul- } \\
\text { tores, proveedores, sub- } \\
\text { contratistas de calidad }\end{array}$ & - \\
\hline
\end{tabular}

Tabla 5 Indicadores por grupos

\begin{tabular}{|c|c|c|c|c|c|c|c|c|c|c|c|}
\hline \multirow{2}{*}{\multicolumn{2}{|c|}{ Indicadores }} & \multicolumn{5}{|c|}{ Grupo I } & \multicolumn{5}{|c|}{ Grupo II } \\
\hline & & A & G & I & $\mathrm{F}$ & $\mathrm{H}$ & $\mathrm{M}$ & $\mathrm{C}$ & $\mathrm{L}$ & $\mathrm{P}, \mathrm{Q}, \mathrm{J}$ & $\mathrm{N}$ \\
\hline \multirow{7}{*}{ 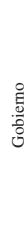 } & Apoyo al emprendimiento & $-1,5$ & $-2,3$ & 1,5 & $-1,6$ & 0,8 & $-2,9$ & $-3,3$ & $-2,6$ & $-1,2$ & 0,7 \\
\hline & Regulaciones no son barrera & 1,2 & 0,8 & 4,0 & 2,2 & $-0,9$ & 1,1 & $-0,2$ & 0,2 & $-0,3$ & 0,0 \\
\hline & $\begin{array}{l}\text { Políticas favorecen la creación de } \\
\text { emprendimiento }\end{array}$ & $-0,4$ & $-1,6$ & 1,4 & $-2,4$ & $-2,3$ & $-1,1$ & $-1,3$ & $-0,8$ & $-1,6$ & $-1,3$ \\
\hline & Efectividad de Burocracia & 1,9 & 1,4 & 2,7 & 2,3 & $-0,1$ & $-0,8$ & 1,0 & 1,0 & 0,8 & 0,7 \\
\hline & Programas para el emprendimiento & $-1,0$ & $-2,6$ & 0,2 & $-3,4$ & $-0,4$ & $-2,2$ & $-3,2$ & $-1,6$ & $-1,7$ & $-1,3$ \\
\hline & Obtención de información & $-0,9$ & $-2,5$ & 3,5 & $-2,9$ & 0,9 & $-2,8$ & $-3,2$ & $-2,6$ & $-1,2$ & $-0,7$ \\
\hline & $\begin{array}{l}\text { Eficiencia Profesionales Parte Gu- } \\
\text { bernamental }\end{array}$ & 0,1 & $-1,2$ & 1,8 & $-0,6$ & 1,3 & $-1,1$ & $-3,3$ & $-0,6$ & $-1,4$ & 1,3 \\
\hline \multirow{4}{*}{ 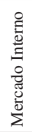 } & Mercado $\mathrm{B} \& \mathrm{~S}$ de consumo & 0,2 & $-0,1$ & $-2,4$ & 1,4 & 0,3 & 0,0 & 0,5 & $-0,6$ & 0,1 & $-0,7$ \\
\hline & $\begin{array}{l}\text { Mercado B\&S de consumo para } \\
\text { empresas }\end{array}$ & $-0,2$ & $-1,0$ & $-0,5$ & 1,6 & 0,3 & $-0,5$ & $-0,5$ & $-1,4$ & $-2,2$ & $-1,3$ \\
\hline & Accesibilidad a nuevos mercados & 0,3 & $-0,5$ & $-0,5$ & $-1,2$ & $-0,9$ & 2,0 & $-0,7$ & 0,8 & 0,7 & 0,0 \\
\hline & Ley antimonopolio & 0,3 & $-1,1$ & $-2,4$ & $-0,7$ & $-1,6$ & 2,8 & $-1,8$ & 2,0 & $-2,6$ & 0,7 \\
\hline \multirow{3}{*}{ 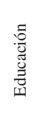 } & $\begin{array}{l}\text { Espíritu Empresarial Educación } \\
\text { Primaria y Secundaria }\end{array}$ & 0,3 & 0,7 & 2,5 & $-2,4$ & 0,8 & $-1,4$ & $-0,3$ & $-1,2$ & $-0,5$ & 2,7 \\
\hline & Presencia de Universidades & $-3,1$ & $-2,8$ & $-0,4$ & $-3,0$ & $-0,6$ & $-3,4$ & $-2,5$ & $-0,8$ & 2,5 & $-1,3$ \\
\hline & $\begin{array}{l}\text { Formación en dirección, gestión de } \\
\text { empresas }\end{array}$ & $-3,4$ & $-3,1$ & 1,9 & $-1,6$ & $-0,3$ & $-3,8$ & $-3,3$ & $-1,8$ & 1,3 & $-1,3$ \\
\hline
\end{tabular}


Tabla 7 Indicadores por grupos

\begin{tabular}{|c|c|c|c|c|c|c|c|c|c|c|c|}
\hline \multirow{2}{*}{\multicolumn{2}{|c|}{ Indicadores }} & \multicolumn{5}{|c|}{ Grupo I } & \multicolumn{5}{|c|}{ Grupo II } \\
\hline & & A & G & I & $\mathrm{F}$ & $\mathrm{H}$ & $\mathrm{M}$ & $\mathrm{C}$ & $\mathrm{L}$ & $\mathrm{P}, \mathrm{Q}, \mathrm{J}$ & $\mathrm{N}$ \\
\hline \multirow{3}{*}{ 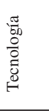 } & Cantidad de Nuevas Tecnologías & $-1,4$ & $-0,6$ & $-3,3$ & $-2,0$ & 0,1 & 0,0 & 0,2 & $-0,8$ & $-1,7$ & 1,3 \\
\hline & Subvenciones Nuevas Tecnologías & $-1,5$ & $-1,9$ & $-2,0$ & $-4,0$ & $-0,9$ & $-0,9$ & $-3,3$ & $-1,8$ & $-0,9$ & $-1,3$ \\
\hline & $\begin{array}{l}\text { Transferencia de tecnología y cono- } \\
\text { cimiento }\end{array}$ & $-2,2$ & $-1,4$ & 1,2 & $-2,2$ & 0,5 & 0,9 & $-3,2$ & $-0,8$ & 2,1 & 0,0 \\
\hline \multirow{3}{*}{ 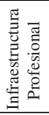 } & $\begin{array}{l}\text { Cantidad de Asesores y Proveedo- } \\
\text { res }\end{array}$ & $-0,5$ & 1,4 & $-1,5$ & 0,6 & $-1,4$ & 1,7 & 1,0 & 2,0 & $-1,3$ & 0,0 \\
\hline & Accesibilidad a servicios bancarios & 0,2 & 1,6 & 0,2 & 0,0 & $-1,6$ & 0,9 & $-0,7$ & 2,0 & $-3,5$ & $-1,3$ \\
\hline & Costo de Asesores y Proveedores & $-0,2$ & 0,2 & $-2,1$ & 1,6 & $-1,3$ & $-0,1$ & 0,0 & $-0,8$ & $-2,8$ & 0,7 \\
\hline \multirow{3}{*}{ 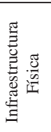 } & Infraestructura física & 1,3 & 2,7 & $-2,1$ & 3,7 & $-0,4$ & 1,1 & 3,5 & 3,2 & $-1,1$ & $-0,7$ \\
\hline & $\begin{array}{l}\text { Acceso a Servicios Telecomunica- } \\
\text { ciones y Básicos }\end{array}$ & 0,6 & 1,9 & 1,2 & 2,8 & 0,4 & 1,4 & 1,8 & 1,6 & 1,1 & $-2,7$ \\
\hline & $\begin{array}{l}\text { Costo de Servicios Básicos y de } \\
\text { Telecomunicaciones }\end{array}$ & 1,1 & 1,3 & $-0,8$ & 2,8 & $-0,1$ & 1,8 & 1,0 & 1,2 & $-2,5$ & $-4,0$ \\
\hline \multirow{3}{*}{ 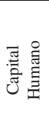 } & $\begin{array}{l}\text { Capacidad de contratar Capital Hu- } \\
\text { mano Necesario }\end{array}$ & 1,3 & 1,5 & $-0,5$ & 3,8 & $-0,9$ & 2,3 & 0,5 & 2,6 & $-1,3$ & $-3,3$ \\
\hline & Capital Humano Capacitado & 1,2 & 2,0 & 2,1 & 1,7 & $-0,4$ & 2,8 & 0,8 & 2,4 & $-1,6$ & $-0,7$ \\
\hline & $\begin{array}{l}\text { Políticas laborales como barrera pa- } \\
\text { ra contratar }\end{array}$ & $-2,8$ & $-2,7$ & $-0,9$ & $-3,6$ & 1,3 & $-4,0$ & $-2,2$ & $-2,6$ & $-0,3$ & 1,3 \\
\hline \multirow{2}{*}{$\frac{\pi}{\tilde{z}}$} & Valor del éxito individual & 0,1 & 0,2 & $-0,2$ & $-1,0$ & $-0,8$ & 0,2 & 0,3 & $-0,4$ & 0,7 & 1,3 \\
\hline & $\begin{array}{l}\text { Estimulan Creatividad, Riesgo em- } \\
\text { presarial e Innovación }\end{array}$ & $-0,3$ & $-0,2$ & 1,0 & $-1,0$ & 0,8 & 0,3 & $-0,5$ & $-1,4$ & $-1,6$ & 2,0 \\
\hline
\end{tabular}

\section{Conclusiones}

Es concluyente, que en la región de estudio, el área gubernamental no prioriza el nacimiento y desarrollo de emprendimientos, de la misma manera los impuestos y burocracia no representan un peso para los emprendimientos ya que su carga no es excesiva y los plazos-tiempos son razonables; en cuanto a los programas que desarrolla el estado son escasos, poco efectivos y de información restringida; no existe capacitación referente a la dirección y administración de emprendimientos por parte del gobierno o entidades privadas, donde las normas sociales no valoran adecuadamente el éxito individual. Ni estimulan lo suficiente la creatividad o asunción del riesgo empresarial.

En el área de estudio las escuelas y colegios apenas estimulan el espíritu empresarial mientras que las universidades, no tienen presencia en cuanto a la educación de profesionales competitivos y desarrollo de proyectos con la sociedad.

Por otra parte, el acceso a tecnologías es restringido, la transferencia de conocimiento entre empresa-universidad es ineficiente. Existe el adecuado capital humano fundamental para la realización normal de las actividades del emprendimiento sin embargo, las políticas laborales representan una gran barrera a la hora de contratar dicho personal.

Existe acceso a una infraestructura física y servicios de telecomunicaciones-básicos para el desarrollo del emprendimiento, sin embargo los costos por estos servicios son significativamente altos para la capacidad de pago del emprendedor, de la misma manera, hay gran accesibilidad a consultores y proveedores, pero no cumplen las expectativas y abastecimientos necesarios para el emprendedor. En cuanto a entidades bancarias estas cubren las necesidades más básicas del emprendedor.

Los precios en los mercados de B\&S (Bienes y Servicios) de consumo así como los mercados de $B \& S$ de consumo para empresa no registran cambios excesivamente radicales de un ejercicio a otro. Mientras que el acceso de nuevas empresas al mercado es medianamente difícil. Además la legislación de antimonopolio es poco efectiva en su aplicación y conocimiento.

Dados los resultados, es esencial desarrollar nexos entre entidades públicas para facilitar la investigación, facilitar acceso a base de datos actualizados y que representen adecuadamente la realidad. Promover capacitaciones efectivas y constantes, por parte de entidades públicasprivadas que permitan la creación y desarrollo de empresas competitivas. Establecer relaciones multilaterales entre los centros educativos y las universidades para desarrollar instrumentos o proyectos para la educación en principios económicos, administrativos y el espíritu empresarial. Lo cual permitirá Potenciar los profesionales de distintas áreas para la mejora de las consultoras en la región.

\section{Referencias Bibliográficas}

Abad, J. (2015). Infraestructura inteligente: La tecnología al servicio de la conectividad física y el transporte. Integración \& comercio(39 (Septiembre)), 96-107.

Abarca, A., Espinoza, J., Mandakovic, V., Rojas, P., y Yañez, C. (2016). GLOBAL ENTREPRENEURSHIP MONITOR REPORTE REGIONAL BIOBÍO 20142015., 73.

Alzate, S. A. (2011, jan). Las políticas públicas en Colombia. Insuficiencias y desafíos. Forum. Revista Departamento Ciencia Política, 1(1), 95-111.

Amorós, J. E. (2011). El proyecto Global Entrepreneurship Monitor (GEM): una aproximación desde el contexto latinoamericano. Academia. Revista Latinoamericana de Administración(46), 1-15.

Amorós, J. E., y Abarca, A. (2014). Global Entrepreneurship Monitor. , 98.

Aragón, B. B. R., Reyes, M. A. C., y Flores, R. M. (2017, jul). Emprendimiento e innovación como detonadores de negocios competitivos en el mercado de 
servicios. Mercados y Negocios (2594-0163), O(36) 21-44.

Barrado, B., y Molina, J. A. (2015). Factores macroeconómicos que estimulan el emprendimiento. , 51.

de Soto, F. C. (2006). La gestión de recursos humanos en las organizaciones de servicio. Laurus, 12(Ext), $10-27$.

Donneys González, F., y Blanco Campins, B. (2016, dec). La transferencia de tecnología en universidades colombianas. Economía y Desarrollo, 157(2), 182198.

Gavilanes, R. V. (2010, mar). Hacia una nueva definición del concepto "política pública". Desafíos, 20(0), 149-187.

León, D. N. (2015, nov). Antecedentes de la configuración de políticas de financiamiento a las pymes. Sistema financiero ecuatoriano y el acceso a financiamiento de las Pymes, 1, 8-6.

Proaño, L. F. H. (2014). La Cultura del Emprendimiento y su Formación. Alternativas, 15(1), 46-50.

Ripol, J. C. (1995). Factores de cambio en la distribución comercial. Derecho privado y Constitución(5), 6784.
Salinas, S., Machado, Ó., Ordoñez, D., y Aquino, I. (2016, jun). Políticas del Estado ecuatoriano y su relación con el desarrollo del emprendimiento. Yachana Revista Científica, 1(1).

Serida, J., Guerrero, C., Alzamora Ruiz, J., Borda, A., y Morales, O. (2017). Global Entrepreneurship Monitor Perú 2016-2017. Lima: Universidad ESAN.

Recibido: 30 de junio de 2018

Aceptado: 3 de septiembre de 2018 
